\title{
Correction to: Robot-assisted laparoscopic reconstructed management of multiple aneurysms in renal artery primary bifurcations: a case report and literature review
}

Hai-bin Wei ${ }^{1 \dagger}$, Xiao-long $\mathrm{Qi}^{{ }^{1 \dagger}}$, Feng $\mathrm{Liu}^{1 *}$, Jie Wang ${ }^{2}$, Xiao-feng $\mathrm{Ni}^{3}$, Qi Zhang ${ }^{1}$, En-hui Li ${ }^{1}$, Xuan-yu Chen ${ }^{1}$ and Da-hong Zhang ${ }^{1 *}$

\section{Correction}

After publication of this work [1] it was noticed the author - Jie Wang's name was in the wrong order.

The original article was corrected.

The publisher apologises for this error.

\section{Author details}

'Department of Urology, Zhejiang Provincial People's Hospital, No. 158, Shangtang Road, Xiacheng District, Hangzhou, Zhejiang 310014, China.

${ }^{2}$ Department of Nephrology, Sir Run Run Shaw Hospital, No. 3, East Qingchun Road, Jianggan District, Hangzhou, Zhejiang 310076, China. ${ }^{3}$ Department of general surgery, Central Hospital of Huzhou, No. 198, Hongqi Road, Wuxing District, Huzhou, Zhejiang 313003, China.

Received: 26 October 2017 Accepted: 26 October 2017

Published online: 03 November 2017

\section{Reference}

1. Wei H-B, Qi X-L, et al. Robot-assisted laparoscopic reconstructed

management of multiple aneurysms in renal artery primary bifurcations: a

case report and literature review. BMC Urol. 2017;17:96.

*Correspondence: liufeng2408@sina.com; zhangdahong88@yeah.net

The online version of the original article can be found under doi:10.1186/ s12894-017-0291-6

${ }^{\dagger}$ Equal contributors

'Department of Urology, Zhejiang Provincial People's Hospital, No. 158,

Shangtang Road, Xiacheng District, Hangzhou, Zhejiang 310014, China 